
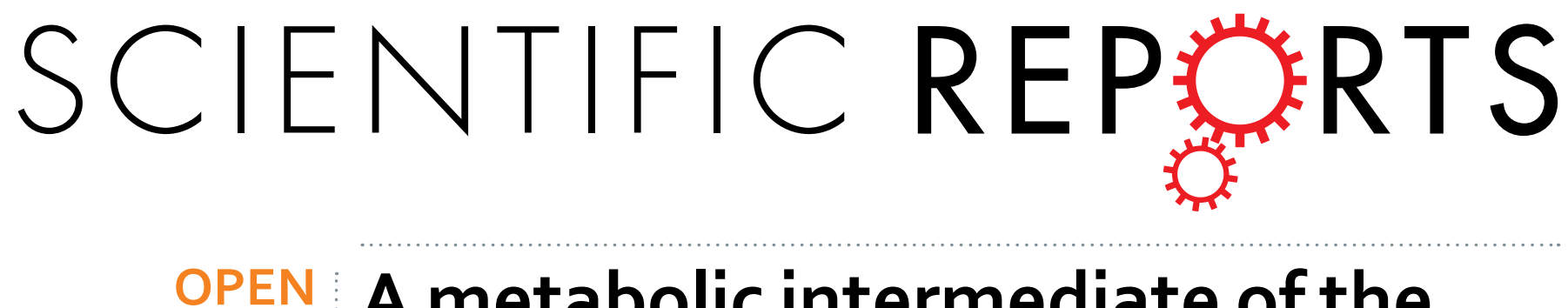

\title{
A metabolic intermediate of the fructose-asparagine utilization pathway inhibits growth of a Salmonella fraB mutant
}

Received: 18 March 2016

Accepted: 27 May 2016

Published: 12 July 2016
Anice Sabag-Daigle ${ }^{1,2}$, Henry M. Blunk ${ }^{3}$, Anindita Sengupta ${ }^{4}$, Jikang Wu ${ }^{4}$, Alexander J. Bogard ${ }^{4}$, Mohamed M. Ali ${ }^{2,3,5}$, Christopher Stahl ${ }^{3}$, Vicki H. Wysocki ${ }^{4}$, Venkat Gopalan ${ }^{4}$, Edward J. Behrman ${ }^{4}$ \& Brian M. M. Ahmer ${ }^{1,2,3}$

Insertions in the Salmonella enterica fra locus, which encodes the fructose-asparagine (F-Asn) utilization pathway, are highly attenuated in mouse models of inflammation ( $>1000$-fold competitive index). Here, we report that $\mathrm{F}$-Asn is bacteriostatic to a fraB mutant $\left(\mathrm{IC}_{50} 19 \mu \mathrm{M}\right)$, but not to the wildtype or a fra island deletion mutant. We hypothesized that the presence of FraD kinase and absence of FraB deglycase causes build-up of a toxic metabolite: 6-phosphofructose-aspartate (6-P-F-Asp). We used biochemical assays to assess $\mathrm{FraB}$ and FraD activities, and mass spectrometry to confirm that the fraB mutant accumulates 6-P-F-Asp. These results, together with our finding that mutants lacking $f r a D$ or the $f r a$ island are not attenuated in mice, suggest that the extreme attenuation of a fraB mutant stems from 6-P-F-Asp toxicity. Salmonella FraB is therefore an excellent drug target, a prospect strengthened by the absence of the fra locus in most of the gut microbiota.

There are over 2600 serovars of Salmonella that can be divided into two pathovars, the gastrointestinal pathovar that causes inflammatory diarrhea, and the extraintestinal pathovar that typically fails to elicit diarrhea but instead causes a systemic infection, Typhoid Fever ${ }^{1}$. Salmonella enterica serovar Typhimurium is the model gastrointestinal serovar, while serovar Typhi is the classic example of an extraintestinal serovar. The gastrointestinal serovars of Salmonella are among the most common of foodborne illnesses in the United States, and are the leading cause of hospitalization and death ${ }^{2}$. Globally, the gastrointestinal serovars are thought to cause 155,000 deaths per year ${ }^{3,4}$. There are no vaccines for human use that protect against the gastrointestinal serovars ${ }^{5,6}$. Antibiotics are used to treat the very young or elderly, or when there are complications or systemic disease. However, for uncomplicated cases of Salmonella-mediated gastroenteritis, antibiotics are not recommended because the disruption to the normal microbiota may actually benefit Salmonella and increase shedding ${ }^{7-9}$. Therefore, drugs that specifically target Salmonella without disrupting the microbiota would be extremely useful. Focusing on the unique metabolic capabilities of this pathogen is likely to prove productive.

Serovar Typhimurium causes inflammation using its two Type 3 Secretion Systems, T3SS1 and T3SS2, encoded within Salmonella Pathogenicity Islands 1 and 2 (SPI1 and SPI2), respectively ${ }^{10}$. This inflammation, which disrupts the microbiota and presumably removes competitors for nutrients ${ }^{11}$, leads to the oxidation of thiosulfate to tetrathionate that can be used by Salmonella as a respiratory electron acceptor ${ }^{12}$. Salmonella then respires while its competitors ferment, allowing Salmonella to grow rapidly ${ }^{13,14}$. Respiration also increases the number of compounds that can be utilized as nutrients, as some (e.g., ethanolamine) can be respired but not fermented ${ }^{15}$.

We recently reported that Salmonella can utilize fructose-asparagine (F-Asn) as a sole carbon and nitrogen source ${ }^{16}$. A fraB::kan mutant of Salmonella was not able to utilize F-Asn and was extremely attenuated in mouse

${ }^{1}$ Department of Microbial Infection and Immunity, The Ohio State University, Columbus, OH 43210, USA. ${ }^{2}$ Center for Microbial Interface Biology, The Ohio State University, Columbus, OH 43210, USA. ${ }^{3}$ Department of Microbiology, The Ohio State University, Columbus, $\mathrm{OH} 43210$, USA. ${ }^{4}$ Department of Chemistry and Biochemistry, The Ohio State University, Columbus, OH 43210, USA. ${ }^{5}$ Department of Medical Microbiology and Immunology, Faculty of Medicine, Mansoura University, Mansoura, Egypt. Correspondence and requests for materials should be addressed to B.M.M.A. (email: ahmer.1@osu.edu) 


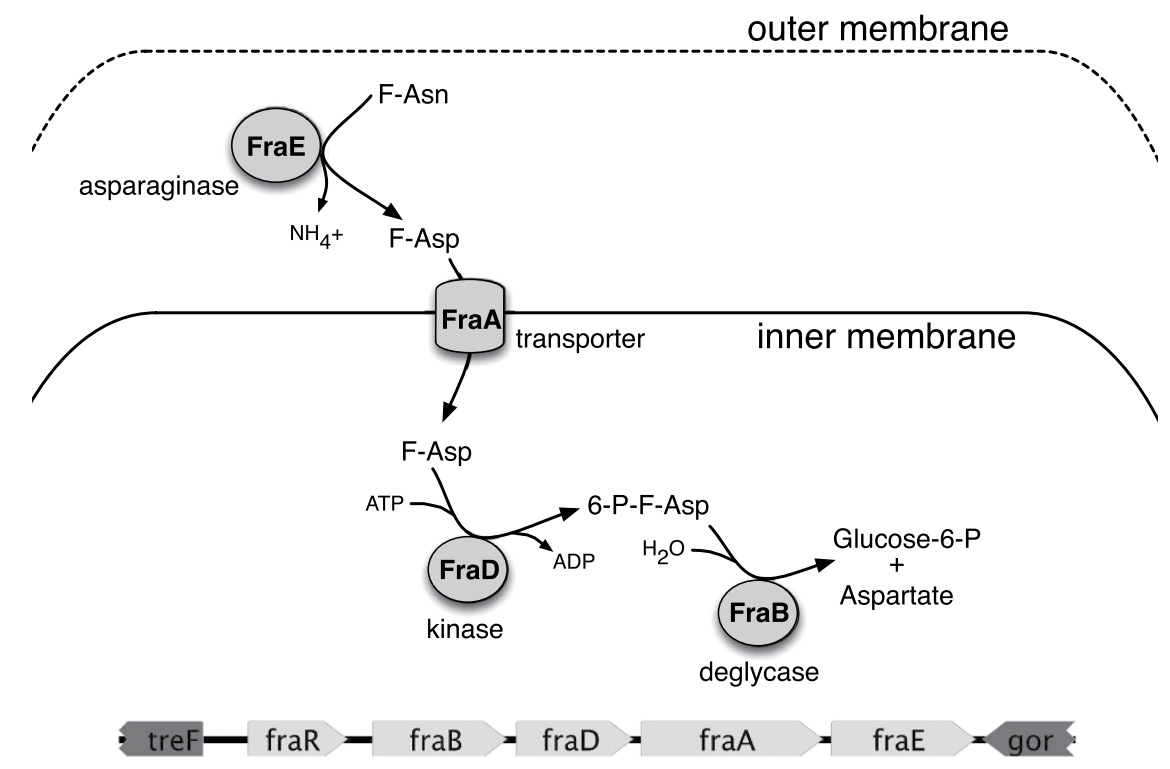

Figure 1. Proposed pathway for fructose-asparagine (F-Asn) utilization in Salmonella enterica and genetic organization of the fra island. The five fra genes are unique to Salmonella while the surrounding treF and gor genes are conserved between E. coli and Salmonella. A proteomic survey of subcellular fractions determined that FraE is periplasmic while FraB is cytoplasmic ${ }^{33}$. The functions of FraD and FraB were proposed based on homology to the E. coli FrlD and FrlB proteins involved in fructose-lysine metabolism ${ }^{18}$, and the inability of a fraB mutant to grow on F-Asn ${ }^{16}$. In this report, we confirm that 6-phosphofructose-aspartate (6-P-F-Asp) is the product of FraD and the substrate of FraB.

models of inflammation ${ }^{16}$. fraB is the first gene of the putative fraBDAE operon. The fraR gene, which encodes a transcription factor of the GntR family, is located upstream of fraBDAE and oriented in the same direction. These five genes make up a horizontally acquired island that is not present in Escherichia coli. The proposed metabolic pathway catalyzed by these gene products is shown in Fig. 1. In this report, we mutated the four structural genes and found that each plays a role in F-Asn utilization, although the fraE mutation did not completely eliminate F-Asn utilization. During these studies we determined that F-Asn is inhibitory to a fraB mutant, but not to the other mutants, and determined that a metabolic intermediate accumulates in the fraB mutant. We found that this inhibition is responsible for the extreme attenuation in mouse models, and identify FraB as a novel drug target.

\section{Results \\ A fraB mutant of Salmonella cannot grow on glucose in the presence of F-Asn. Wild-type Salmonella and an isogenic fraB mutant grow equally well on glucose as sole carbon source (Fig. 2). The wild-type grows on F-Asn as the sole carbon source, while the $f r a B$ mutant does not. Surprisingly, we found that the fraB mutant does not grow when both F-Asn and glucose are provided together (Fig. 2). We found that the lack of growth on the dual carbon source medium is specific to the fraB mutant. Mutants lacking fraD, fraE, fraA, or the entire fra island, grow as well as the wild-type in medium containing both F-Asn and glucose (Fig. 2). Because a fraD mutation does not have this phenotype, and it encodes the enzyme just upstream of FraB in the pathway, we hypothesized that the putative metabolic intermediate, 6-phosphofructose-aspartate (6-P-F-Asp), is toxic to the cell (Fig. 2). Deletion of both $f r a B$ and $f r a D$ simultaneously or fraB, fraD, and fraA, relieved the toxicity, consistent with the need for FraD to produce the toxic intermediate (Fig. 2).}

6-P-F-Asp accumulates in the fraB mutant. To test the idea that F-Asn toxicity results from the presence of FraD and absence of FraB, we adopted an approach that combined enzymatic assays and mass spectrometry. We first used biochemical assays to measure FraD and FraB activities in crude lysates prepared from wild-type Salmonella and in mutants lacking either fraB, fraD or the entire fra island, all grown for a short duration in $5 \mathrm{mM}$ F-Asn (see Methods). These measurements were made possible due to in-house synthesis of F-Asp and 6-P-F-Asp, the substrates of FraD and FraB, respectively ${ }^{17}$, and design of coupled assays that enabled facile colorimetric readouts (Sengupta and Gopalan, manuscript in preparation). Comparing the wild type and fra island deletion mutant offers a positive and negative control cohort (Table 1). In the $\Delta f r a B:$ :kan mutant, there is near-wild-type FraD but no FraB activity (Table 1). In the fraD mutant, there is no FraD activity, and the FraB activity is two-thirds of that observed in the wild type (Table 1); it is possible that deletion of fraD led to some modest regulatory changes that dampened FraB expression. Regardless, the overall trends are as expected.

We next employed mass spectrometry (MS) to measure 6-P-F-Asp levels in the wild type and fra mutants. An aliquot of the cells used for the enzyme assays (described above) were used for the MS measurements. 6-P-F-Asp was detected in the $f r a B$ mutant, but was not detected in the wild-type or the fra island deletion mutant (Fig. 3, 
A

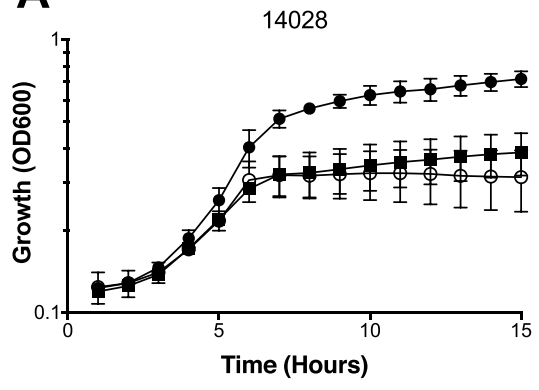

D

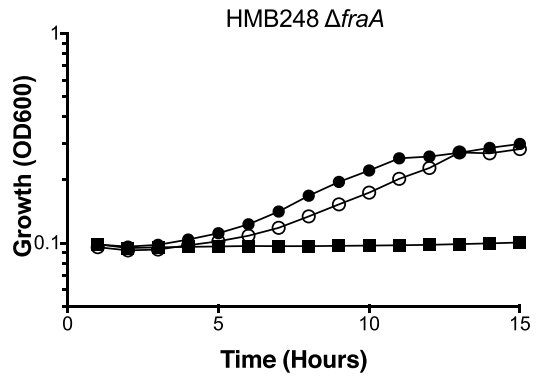

G

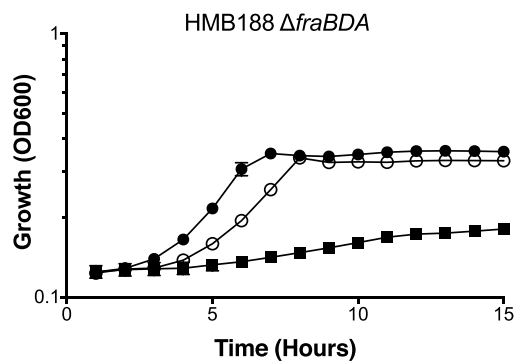

B

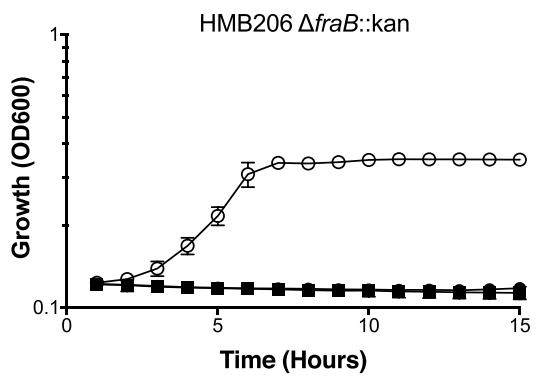

E

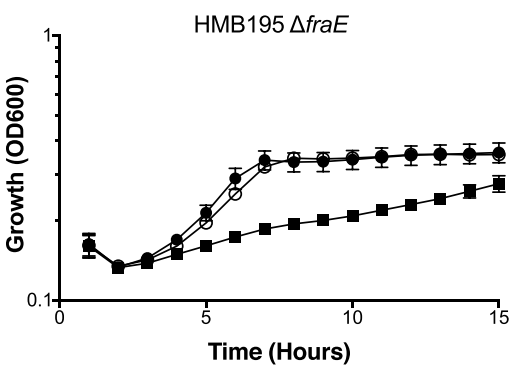

H

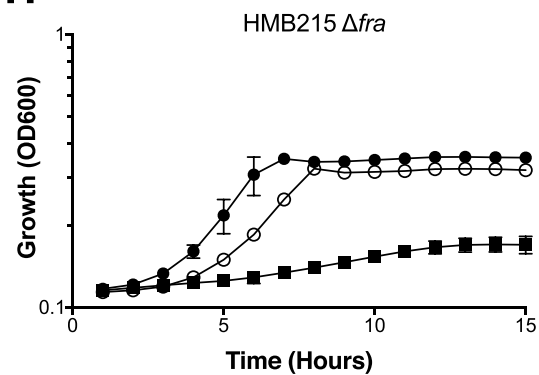

C

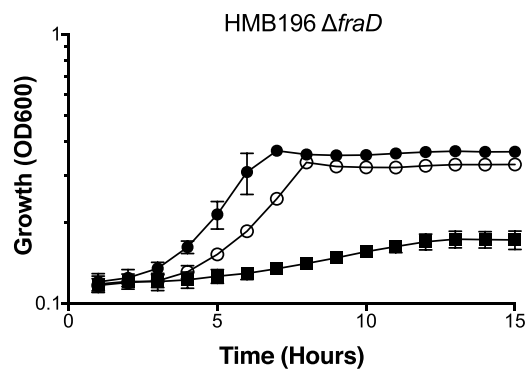

$\mathbf{F}$

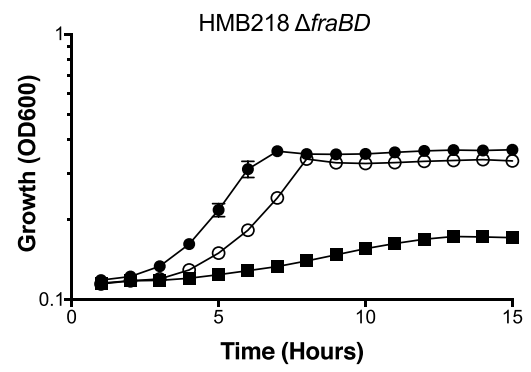

Figure 2. Growth of fra mutants on different carbon sources. Wild-type Salmonella (14028) and isogenic fra mutants were grown in M9 minimal media with $\sim 19 \mathrm{mM}$ ammonium chloride supplemented with either $5 \mathrm{mM}$ glucose (open circles), $5 \mathrm{mM}$ F-Asn (closed squares), or $5 \mathrm{mM}$ glucose and $5 \mathrm{mM}$ F-Asn (closed circles). All data points are the mean of three biological replicates measured in triplicate ( 9 total points). Error bars represent standard deviation.

\begin{tabular}{|l|c|c|}
\hline & FraD $(\times \mathbf{1 0} \mathbf{4} / \mathbf{m g})$ & FraB $\left(\times \mathbf{1 0} \mathbf{4}^{\mathbf{U}} / \mathbf{m g}\right)$ \\
\hline Wild-type & $2.4 \pm 0.13$ & $1.3 \pm 0.16$ \\
\hline$\Delta$ fraB::kan & $2.2 \pm 0.15$ & $\mathrm{ND}$ \\
\hline$\Delta$ fraD & $\mathrm{ND}$ & $0.9 \pm 0.06$ \\
\hline$\Delta$ fra & $\mathrm{ND}$ & $\mathrm{ND}$ \\
\hline
\end{tabular}

Table 1. Specific activities of FraD (kinase) and FraB (deglycase) in Salmonella wild-type and mutant extracts. The specific activities reported are the mean and standard deviation values from three independent experiments (see Methods); ND - not detectable.

Supplementary Figs 1-3, Supplementary Tables 1-4). Collectively, results from the biochemical assays and mass spectrometry studies establish that the product generated by FraD is 6-P-F-Asp, which accumulates in the absence of FraB, as would be expected from the predicted pathway for F-Asn utilization (Fig. 1).

F-Asn is bacteriostatic, not bactericidal. To determine if F-Asn is bacteriostatic or bactericidal to the fraB mutant, we determined the $50 \%$ and $90 \%$ inhibitory concentration $\left(\mathrm{IC}_{50}\right.$ and $\left.\mathrm{IC}_{90}\right)$ and the minimum bactericidal concentration $(\mathrm{MBC})$. $\mathrm{The}^{\mathrm{IC}_{50}}$ and $\mathrm{IC}_{90}$ were determined by adding increasing concentrations of $\mathrm{F}$-Asn to M9 minimal medium containing glucose as the carbon source. Growth of the fraB mutant was then monitored in these media. F-Asn did not affect the wild-type or fra island mutant but was inhibitory to growth of the fraB mutant with an $\mathrm{IC}_{50}$ of $19 \mu \mathrm{M}$ ( $95 \%$ confidence interval of 9 to $\left.40 \mu \mathrm{M}\right)$ and $\mathrm{IC}_{90}$ of $174 \mu \mathrm{M}(95 \%$ confidence interval of 82 to $368 \mu \mathrm{M})$ (Fig. 4). To determine the MBC, we removed a small aliquot of these same cultures and performed dilution plating onto LB agar. Such an approach helps determine if the bacteria can recover from the inhibition and grow 


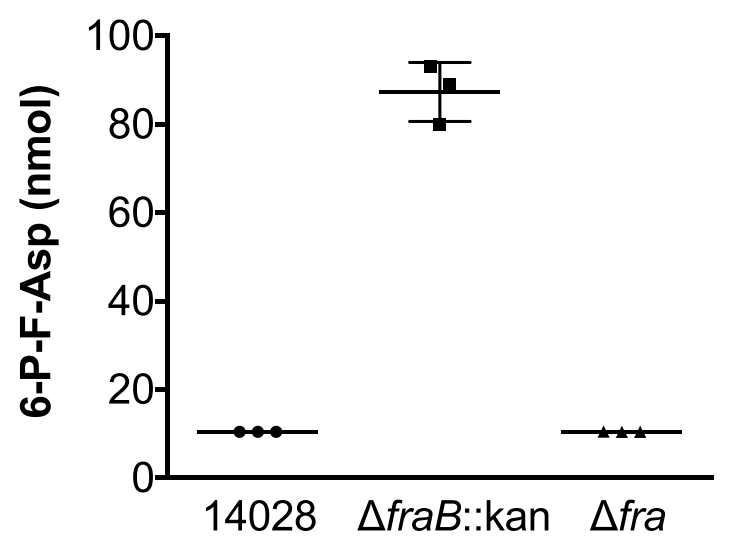

Figure 3. Each point represents mass spectrometry (MS)-based measurement of the levels of 6-P-F-Asp in one biological replicate. Samples were prepared using the cell pellet from a 1.3-ml culture of Salmonella wild-type (14028) or $\Delta f r a B:: k a n(H M B 206)$ or $\Delta f r a$ island deletion (HMB215) mutant. Two transitions were measured to confirm quantitation; data from one transition (Table S4B) are shown here, while data from a second transition (Table S4A) are shown in Fig. S3. The values provided are the mean \pm standard deviation from three biological replicates. The values for wild-type and $\Delta f r a$ island deletion are very low and below $20 \mathrm{nmol}$, the lowest concentration used for establishing the standard curve; however, they are provided here to indicate the reproducible absence of 6-P-F-Asp in these two strains.

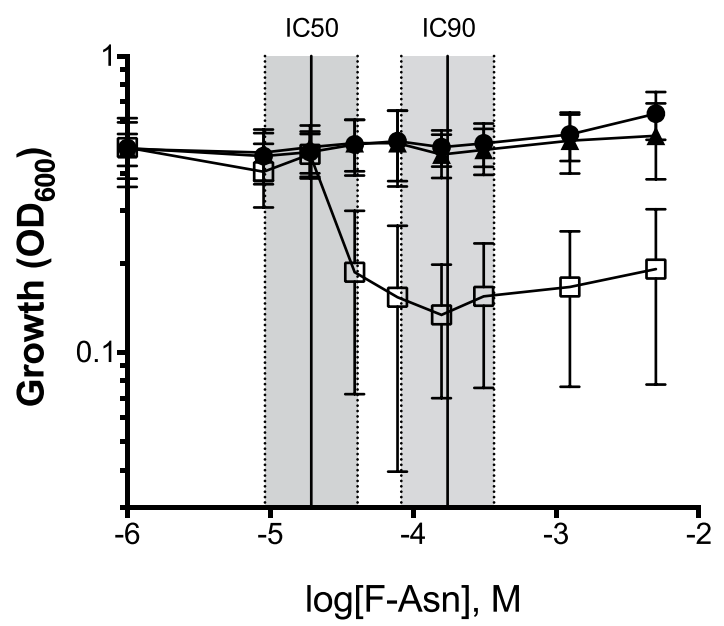

Figure 4. Determination of the $\mathrm{IC}_{50}$ and $\mathrm{IC}_{90}$ of F-Asn to a fraB mutant. Wild-type (14028, closed circles), $\Delta f r a B:: k a n$ (HMB206, open squares) or $\Delta$ fra island (HMB205, closed triangles) were grown in M9 minimal medium containing $5 \mathrm{mM}$ glucose and varying concentrations of F-Asn. The data points represent the optical density obtained after $15 \mathrm{~h}$ of growth at $37^{\circ} \mathrm{C}$ (three biological replicates with three technical replicates in each (nine total replicates); error bars represent standard deviation. $\mathrm{IC}_{50}$ and $\mathrm{IC}_{90}$ were calculated and plotted \pm the $95 \%$ confidence intervals. The $\mathrm{IC}_{50}$ is $19 \mu \mathrm{M}(9$ to $40 \mu \mathrm{M})$ and the $\mathrm{IC}_{90}$ is $174 \mu \mathrm{M}$ (82 to $\left.368 \mu \mathrm{M}\right)$.

on a non-inhibitory medium. Since Salmonella could recover even at the highest concentrations of F-Asn tested, we could not obtain an MBC. Moreover, these results indicate that the inhibition experienced by a fraB mutant grown on F-Asn is bacteriostatic and not bactericidal.

The phenotype of a fraB mutant in mice is due to the toxicity of F-Asn. We previously determined that a fraB mutant had a severe fitness defect (up to 100,000-fold) using competition assays in several mouse models of inflammation, and interpreted this result as a reflection of the importance of F-Asn as a critical nutrient for Salmonella in the inflamed intestine ${ }^{16}$. The realization that a fraB mutant is inhibited by low concentrations of F-Asn (Fig. 2) provides an alternative explanation based on toxicity of 6-P-F-Asp for the fraB fitness defect in mice. To distinguish between these two mechanisms, we tested a fraB mutant, a fraD mutant, and a mutant lacking the entire fra island for fitness in streptomycin-treated Swiss Webster mice (Fig. 5). The expectation was that the fraD and fra island deletion mutants, which are not inhibited by F-Asn (Fig. 2), should help assess the importance of F-Asn as a nutrient. Neither the fraD nor the fra island mutant were attenuated in these mice, while the $f r a B$ mutant was attenuated about 1,000-fold (Fig. 5). Therefore, at least in this streptomycin-treated Swiss Webster mouse model, the entire phenotype of the fraB mutant appears to be due to the toxicity of F-Asn rather than an 
A

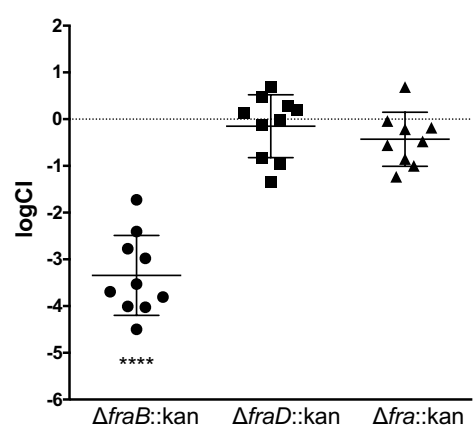

B

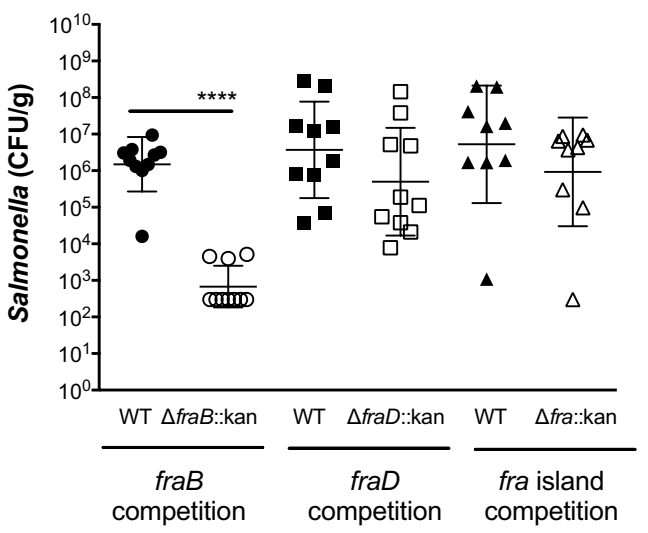

Figure 5. (A) Competitive index (CI) of fra mutants in vivo. Mixtures (1:1) of wild-type JLD1214 and a mutant (either HMB206 $\Delta$ fraB80::kan, HMB184 $\Delta$ fraD4::kan, or HMB205 $\Delta$ fra80::kan) were administered intragastrically (i.g.) to mice that had been treated $24 \mathrm{~h}$ earlier with $20 \mathrm{mg}$ of streptomycin. Mice were monitored for survival and four days post-infection ceca were harvested and plated for CFU. Each point is the $\mathrm{CI}$ from one mouse with the geometric mean and standard deviation shown. Statistical significance for each group being different than 0 was calculated using a one-sample, two-tailed t-test ${ }^{* * * *} \mathrm{p}$-value $\left.<0.0001\right)$. (B) The raw CFU counts that gave rise to the CI were log transformed and plotted (limit of detection of 300 $\mathrm{CFU})$. Error bars show the mean and standard deviation $\left({ }^{* * *} \mathrm{P}<0.0001\right)$.

inability to use F-Asn as a nutrient. The decline of the fraB mutant CFU may suggest that F-Asn is bactericidal rather than bacteriostatic to the mutant in vivo (Fig. 5B). Further studies will be required to test this hypothesis.

\section{Discussion}

This study provides experimental evidence to support a F-Asn catabolic pathway (Fig. 1), which we recently proposed based on the genes in the fra locus and on a knowledge of the enzymes required for utilization of F-Lys, another Amadori compound ${ }^{16,18}$. Validation of metabolic pathways has typically entailed either assessing the growth of genetic mutants under specific limiting conditions or using biochemical and genetic approaches to cause roadblocks at specific stages in a multi-step conversion to identify the metabolite that accumulates. Here, we have employed both strategies.

Mutations in $f r a B$, fraD, and fraA eliminate the ability of Salmonella to grow on F-Asn, while a mutation in fraE reduces growth on F-Asn (Fig. 2). The residual growth of the fraE mutant on F-Asn is likely due to redundancy from other periplasmic asparaginases, a hypothesis that we are testing. Unlike all of the other fra mutants, the fraB mutant has the unusual property of not being able to grow on glucose if F-Asn is also present (Fig. 2). Since FraB is the terminal enzyme in the pathway, we hypothesized that the substrate of FraB may be toxic to the cell. Through the use of mass spectrometry and fraB/fraD mutants, we have confirmed that the FraB substrate is 6-P-F-Asp and that 6-P-F-Asp accumulates in a fraB mutant (Figs 1 and 3). Moreover, the presence of FraB deglycase and FraD kinase activities in wild-type Salmonella crude extracts confirms the postulated route for F-Asn metabolism; the complete absence of FraD and FraB activities in the fra island mutant suggests little functional redundancy with respect to F-Asn utilization (Table 1). Ongoing studies with recombinant Fra enzymes are expected to provide additional insights into the individual biochemical transformations in the F-Asn pathway.

In addition to furthering our understanding of the F-Asn utilization pathway, results from this study revealed an unexpected possibility for inhibiting Salmonella growth with clear implications for future drug discovery. Mice with intact microbiota are highly resistant to Salmonella-mediated inflammation in the gastrointestinal tract. However, disruption of the microbiota by agents such as streptomycin causes the mice to be susceptible to Salmonella-mediated inflammation. Similarly, germ-free mice and IL10-deficient mice are also susceptible. We previously reported that two independently constructed fraB::kan mutants of Salmonella were dramatically attenuated in all of the mouse models that are susceptible to inflammation but not in conventional mice ${ }^{16}$. Because both mutants could be complemented with a plasmid encoding the fra island ${ }^{16}$, we inferred that F-Asn is of utmost importance to Salmonella as a nutrient during growth in the inflamed intestine. However, we have now discovered that the phenotype is not due to the importance of F-Asn as a nutrient but is rather due to the accumulation of an inhibitory metabolite in the F-Asn utilization pathway: 6-P-F-Asp. Several lines of evidence support this claim. First, observations from growth of different fra mutants in minimal medium containing both glucose and F-Asn as carbon sources proved instructive. A mutant lacking fraD, or the entire fra island, can grow in this medium, while a fraB mutant cannot (Figs 2 and 4). Second, mass spectrometric studies demonstrate that 6-P-F-Asp accumulates to high levels in the $\mathrm{fraB}$ mutant, but not in a fra island deletion mutant (Fig. 3). Third, a fraB mutant is extremely attenuated in streptomycin-treated Swiss Webster mice, while a fraD mutant and a mutant lacking the entire fra island have no fitness defect (Fig. 5). Since neither a fraD mutant nor a mutant lacking the entire $\mathrm{fra}$ island can grow on F-Asn, their failure to utilize F-Asn in this particular mouse model does not result in a measurable fitness defect and demonstrates that F-Asn is not an essential nutrient during infection. Last, the organization of the fra operon suggests a strategy adopted by Salmonella to prevent build-up of 
6-P-F-Asp - the fra genes are encoded in the opposite order of the enzymatic pathway (Fig. 1), possibly to ensure that FraB is expressed before FraD thereby avoiding an accumulation of the FraD product.

Except for Salmonella, only a few Citrobacter and Clostridia seem to have the fra genes although there is no experimental evidence for F-Asn utilization by the latter ${ }^{16}$. Thus, FraB represents a potential drug target whose inhibition will selectively affect Salmonella and perhaps a few members of the microbiota. As with numerous other genes involved with anaerobic metabolism in the gut, the fra locus is widely distributed and conserved among the gastrointestinal serovars of Salmonella, but is missing or mutated in serovars of the extraintestinal pathovar (the typhoidal serovars) ${ }^{1}$. Any inhibitor of FraB would only work in the presence of F-Asn. Given the $\mathrm{IC}_{50}$ of F-Asn for a a FraB mutant in vitro is $19 \mu \mathrm{M}$ (in M9 minimal medium containing $5 \mathrm{mM}$ glucose), the amount of F-Asn required for toxicity in vivo is likely to be low and easily met in the diet, as evident from the attenuation of a fraB mutant in mice ${ }^{16}$. The concentration of F-Asn has only been measured in a few human foods, but F-Asn in some vegetables (e.g., asparagus) is as high as $1.4 \%$ of dry weight ${ }^{19,20}$. Since the F-Asn concentration in a human with Salmonellosis would not be known, a FraB inhibitor could be administered with F-Asn to ensure the inhibition of Salmonella. F-Asn would only be available to Salmonella during inflammation. When inflammation is relieved, the microbiota would be restored, and F-Asn levels would decrease. It is probable that loss-of-function mutations within $\mathrm{fraD}$ or $\mathrm{raA}$ would provide resistance to the inhibitor, but selection for these mutants is likely to be brief, and cease when symptoms cease.

Sugar phosphates (e.g., those of rhamnose, glucose, arabinose, and galactose) are known to be toxic to E. coli and Salmonella, in part due to depletion of glycolytic metabolites or biosynthetic precursors ${ }^{21-26}$. While we do not know the basis for 6-P-F-Asp toxicity, the F-Asn catabolic pathway has two major advantages over these other pathways with regard to drug discovery. First, the F-Asn pathway is more specific to Salmonella, so fewer members of the microbiota are likely to be adversely affected by an inhibitor. Second, the toxicity of the glucose and galactose catabolic pathways can be overcome by the addition of other nutrients in vitro ${ }^{21,22,27,28}$. The observation that only the F-Asn utilization pathway was identified in our transposon site hybridization screening in mice, is consistent with the idea that F-Asn toxicity cannot be overcome by the presence of other nutrients that are available in the inflamed intestine ${ }^{16}$. It is also possible that a FraB inhibitor would be bactericidal to Salmonella in vivo rather than bacteriostatic, as reflected by the precipitous decline in the CFU of the fraB mutant from mice (Fig. 5B). Additional experiments are required to test this hypothesis, and the possibility that additional stressors in the inflamed intestine, that are not present in our in vitro assays, combine with 6-PF-Asp toxicity to kill Salmonella in vivo.

\section{Methods}

Strains and Media. Strains used in this study are listed in Table 2. Bacteria were routinely grown in LuriaBertani (LB) broth (EMB) or on LB agar plates made by adding 1.5\% (w/v) agar (Fisher Bioreagents). For growth studies involving F-Asn, we employed M9 minimal medium ${ }^{1,29}: 1 \times \mathrm{M} 9$ salts, $2 \mathrm{mM} \mathrm{MgSO}_{4}, 0.1 \mathrm{mM} \mathrm{CaCl}_{2}$, $0.01 \mathrm{mM}$ thiamine, and trace metals ${ }^{1,30}$. For the growth assays using minimal media without nitrogen, $\mathrm{NH}_{4} \mathrm{Cl}_{2}$ was not included in the $10 \times \mathrm{M} 9$ media. As needed, chloramphenicol (cam, $10 \mu \mathrm{g} / \mathrm{mL})$ or kanamycin $(\mathrm{kan}, 50 \mu \mathrm{g} / \mathrm{mL})$ were added to the media.

Construction of mutants. Lambda Red mutagenesis was used to generate insertion mutations or in-frame deletions of target genes ${ }^{10,31}$. Oligonucleotides containing 40 nucleotides of identity to the target genes were appended to sequences that bind the $\mathrm{P} 1$ and $\mathrm{P} 2$ sites of $\mathrm{pKD} 4$ or $\mathrm{pKD} 3^{11,31}$. Primer sequences used are listed in Table 3. The FRT antibiotic resistance cassette of either pKD3 or pKD4 was amplified by PCR to generate a product with $40 \mathrm{bp}$ identity to target genes on each end. This PCR product was electroporated into strain $14028+$ pKD46, and homologous recombinants were selected using LB kan at $37^{\circ} \mathrm{C}$. Correct insertion of the FRT antibiotic cassette was confirmed by PCR using one primer within the antibiotic cassette and another outside the region of homologous recombination. The bona fide mutants were transduced into 14028 using phage P22HTint. The antibiotic cassette was then removed by electroporating pCP20 $\left(\mathrm{amp}^{\mathrm{r}}\right)$, which encodes FLP recombinase, into the strain and plating on LB amp at $30^{\circ} \mathrm{C}$. Single colonies were streaked onto LB and incubated at $42^{\circ} \mathrm{C}$ to cure the strain of pCP20. PCR was used to verify loss of the antibiotic resistance cassette using primers upstream and downstream of the target gene. Colonies with the correct PCR product were also screened for loss of the antibiotic resistance gene and pCP20 $\left(\mathrm{amp}^{\mathrm{r}}\right)$.

Growth assays. Growth curves were performed using clear, flat-bottom, 96-well plates. Minimal media with the specified carbon source was prepared and overnight cultures were washed twice with sterile water. In each well, $198-\mu \mathrm{l}$ aliquots of media were inoculated with $2 \mu \mathrm{l}$ of washed overnight cultures. A Breathe-Easy membrane film (Diversified Biotech) was placed over the 96 -well plate. Growth over $18 \mathrm{~h}$ at $37^{\circ} \mathrm{C}$ was measured using hourly $\mathrm{OD}_{600}$ measurements in the SpectraMax M5 (Molecular Devices) Microplate Reader and the SoftMax Pro 6.1 software.

Synthesis of fructose-asparagine and 6-phosphofructose-aspartate. We have previously synthesized fructose-asparagine ${ }^{16}$ with the reaction carried out in refluxing methanol. However, the yields were poor due to the low solubility of asparagine in methanol. Prof. Valeri Mossine (University of Missouri) suggested an approach to overcome this solubility problem: dissolve the components in a small amount of water, add glycerol (or ethylene glycol), and finally remove the water by rotary evaporation. Remarkably, with this method, all of the components remain in solution. Glucose $(1 \mathrm{~g}, 5.5 \mathrm{mmol})$ and potassium L-aspartate $(0.2 \mathrm{~g}, 1.2 \mathrm{mmol})$ were dissolved in $3 \mathrm{~mL}$ water. Glycerol $(3 \mathrm{~g})$ was added and the mixture rotary evaporated at $50{ }^{\circ} \mathrm{C}$ to remove the water. The viscous homogeneous solution was heated at $60^{\circ} \mathrm{C}$ in an unstoppered flask for $36 \mathrm{~h}$. Glycerol was removed by dissolution in isopropanol. The precipitated product was dissolved in water and applied to a 


\begin{tabular}{|c|c|c|}
\hline Strains & Genotype & Reference or construction \\
\hline 14028 & $\begin{array}{l}\text { Wild-type Salmonella enterica subspecies } \\
\text { enterica serovar Typhimurium }\end{array}$ & ATCC \\
\hline HMB176 & $14028 \triangle$ fraBDA80::cam & $\begin{array}{l}\text { lambda red mutation of fraBDA made using PCR primers BA2553 } \\
\text { and BA2511 and transduced into a clean } 14028 \text { background. }\end{array}$ \\
\hline HMB182 & $14028 \Delta$ fraE4::kan & $\begin{array}{l}\text { lambda red mutation of fraE made using PCR primers BA2537 and } \\
\text { BA2515 and transduced into a clean } 14028 \text { background. }\end{array}$ \\
\hline HMB184 & $14028 \Delta$ fraD4::kan & $\begin{array}{l}\text { lambda red mutation of } \text { fraD made using PCR primers BA2494 and } \\
\text { BA2495 and transduced into a clean } 14028 \text { background. }\end{array}$ \\
\hline HMB188 & $14028 \triangle$ fraBDA80 & Antibiotic cassette in HMB176 was flipped out using pCP20. \\
\hline HMB195 & $14028 \Delta$ fraE4 & Antibiotic cassette in HMB182 was flipped out using pCP20. \\
\hline HMB196 & $14028 \Delta$ fraD4 & Antibiotic cassette in HMB184 was flipped out using pCP20. \\
\hline HMB205 & $14028 \Delta$ fra $80::$ kan & $\begin{array}{l}\text { lambda red mutation of fra island made using PCR primers BA2538 } \\
\text { and BA2513 and transduced into a clean } 14028 \text { background. }\end{array}$ \\
\hline HMB206 & $14028 \Delta$ fraB80::kan & $\begin{array}{l}\text { lambda red mutation of fraB made using PCR primers BA2552 and } \\
\text { BA2553 and transduced into a clean } 14028 \text { background. }\end{array}$ \\
\hline HMB211 & $14028 \triangle$ fraBD81::kan & $\begin{array}{l}\text { lambda red mutation of } f r a B D \text { made using PCR primers BA2553 } \\
\text { and BA2495 and transduced into a clean } 14028 \text { background. }\end{array}$ \\
\hline HMB215 & $14028 \Delta$ fra 80 & Antibiotic cassette in HMB205 was flipped out using pCP20. \\
\hline HMB218 & $14028 \triangle$ fraBD81 & Antibiotic cassette in HMB211 was flipped out using pCP20. \\
\hline HMB247 & $14028 \Delta$ fraA4::kan & $\begin{array}{l}\text { lambda red mutation of fraA made using PCR primers BA2510 and } \\
\text { BA2511 and transduced into a clean } 14028 \text { background. }\end{array}$ \\
\hline HMB248 & $14028 \Delta$ fraA4 & Antibiotic cassette in HMB247 was flipped out using pCP20. \\
\hline JLD1214 & 14028 IG (pagC-STM14_1502)::cam & 16 \\
\hline Plasmids & Description & Reference \\
\hline pKD46 & $\mathrm{P}_{\mathrm{BAD}}$ gam bet exo $\mathrm{pSC} 101$ oriTS $\left(\mathrm{amp}^{\mathrm{r}}\right)$ & 31 \\
\hline pKD3 & FRT-cam-FRT oriR6K $\left(\mathrm{amp}^{\mathrm{r}}\right)$ & 31 \\
\hline pKD4 & FRT-kan-FRT oriR6K $\left(\mathrm{amp}^{\mathrm{r}}\right)$ & 31 \\
\hline pCP20 & cl857 $\lambda$ PR flp pSC101 oriTS $\left(\mathrm{amp}^{\mathrm{r}} \mathrm{cam}^{\mathrm{r}}\right)$ & 34 \\
\hline
\end{tabular}

Table 2. Strains and Plasmids.

Dowex-50 column in the hydrogen ion form as reported for the synthesis of F-Asn ${ }^{16}$. Mass spectrometry in the negative ion mode showed [fructose aspartic acid] ${ }^{-}$at $\mathrm{m} / \mathrm{z} 294(100 \%)$ and [aspartic acid] $^{-}$at m/z $132(40 \%)$. 6-Phosphofructose-aspartate (6-P-F-Asp) could be made only by the glycerol (or ethylene glycol) procedure because the starting material, glucose-6-phosphate, is insoluble in methanol. 6-P-F-Asp was characterized by mass spectrometry and by both proton and ${ }^{13} \mathrm{C} \mathrm{NMR}$, and these details are provided elsewhere ${ }^{17}$.

Preparation of Salmonella extracts. Salmonella were grown in $20 \mathrm{ml} \mathrm{LB}$ for $16 \mathrm{~h}$ at $37^{\circ} \mathrm{C}$ with shaking. The cells were harvested by centrifugation, re-suspended in $20 \mathrm{ml}$ fresh LB supplemented with $5 \mathrm{mM}$ F-Asn, and grown for $30 \mathrm{~min}$ at $37^{\circ} \mathrm{C}$ with shaking. These cells were subjected to two cycles of centrifugation $(5,000 \mathrm{~g}$ at $4^{\circ} \mathrm{C}$ ) and washes with water, before re-suspension in $1 \mathrm{ml}$ of $25 \mathrm{mM}$ HEPES (pH 7.5), $0.1 \mathrm{mM}$ phenylmethylsulfonyl fluoride. Cells were then lysed by sonication ( $50 \%$ output power for $60 \mathrm{~s}$, with cycles of $2 \mathrm{~s}$ on and $5 \mathrm{~s}$ off; Ultrasonic Processor, Cole-Parmer), and debris removed by centrifuging the cell lysate at 13,000 g for $20 \mathrm{~min}$ at $4^{\circ} \mathrm{C}$. At this point the samples were split with half for enzymatic assays and half for mass spectrometry measurements. For enzymatic assays, after addition of $0.1 \mathrm{mg} / \mathrm{ml} \mathrm{BSA}$, the supernatant was dialyzed against $25 \mathrm{mM}$ HEPES ( $\mathrm{pH} 7.5$ ) at $4{ }^{\circ} \mathrm{C}$ with two changes over $60 \mathrm{~min}$. The crude dialysates were used for the activity assays described below. To calculate specific activities, the protein content in the crude dialysates was determined using the Bradford assay ${ }^{32}$, with bovine serum albumin serving as the standard.

Enzyme assays. All assays ( $40 \mu \mathrm{l}$ volume) were carried out at $37^{\circ} \mathrm{C}$. For the measurement of FraB deglycase activity, we used a glucose-6-phosphate dehydrogenase (G6PD)-based coupled assay. The FraB reaction mixture contained $1 \mathrm{mM}$ 6-P-F-Asp, $25 \mathrm{mM}$ HEPES (pH 7.5), $5 \mathrm{mM} \mathrm{MgCl}_{2}, 0.1 \mathrm{mM}$ EGTA, $0.5 \mathrm{mM}$ NADP ${ }^{+}, 0.15$ U G6PD (Sigma, G6378). The reaction was initiated by the addition of a defined amount of crude lysates (up to $40 \%$ of the assay volume) obtained from wild-type or mutant Salmonella strains. The NADPH generated by G6PD was followed by measuring absorbance at $340 \mathrm{~nm}$ and taken as a direct readout of the glucose-6-phosphate produced by FraB. To determine FraD kinase activity, a G6PDH + FraB-based coupled assay was performed. The reaction mixture for the kinase assay contained $1 \mathrm{mM}$ fructose-aspartate (F-Asp), $25 \mathrm{mM}$ HEPES (pH 7.5), $25 \mathrm{mM}$ $\mathrm{KCl}, 1 \mathrm{mM} \mathrm{MgCl}_{2}, 1 \mathrm{mM}$ dithiothreitol, $1 \mathrm{mM}$ ATP, $0.1 \mathrm{mM}$ EGTA, $0.5 \mathrm{mM}$ NADP ${ }^{+}, 0.3 \mu \mathrm{M}$ recombinant FraB (Sengupta and Gopalan, unpublished) and 0.15 U G6PD (Sigma, G6378). For both FraB and FraD assays, the reactions were terminated by addition of $6 \mathrm{mM}$ EDTA (final concentration). One unit of activity is defined as the amount of enzyme catalyzing the formation of $1 \mu \mathrm{mol}$ of NADPH per min. Mean and standard deviation values were calculated from independent assays that used crude lysates from three separate cultures.

Mass spectrometry. To measure intracellular 6-P-F-Asp, the bacteria were grown as described above for preparation of Salmonella extracts and the cell pellets were re-suspended in $20 \mathrm{ml}$ water. The cell suspension was 


\begin{tabular}{|c|c|c|}
\hline Primer & equence & Description \\
\hline BA2494 & ATTGTAAAGACAAACAAGGAATAATGATGATGTGTAGG & \\
\hline BA2495 & TACATTGAGGGACGTAACCTATTGTGCAAACATATGAATATCCTCCTTA & $\begin{array}{l}\text { reverse primer for fraD and fraBD } \\
\text { lambda red mutagenesis }\end{array}$ \\
\hline BA2510 & AGGAGGAAGTATGTTTTGGACGGAATTATGTTTTATCCTTGTGTAGGCTGGAGCTGCTTC & $\begin{array}{l}\text { forward primer for fraA lambda red } \\
\text { mutagenesis }\end{array}$ \\
\hline BA2511 & TGAATAACAATCAGGCCAGAACCATTTTTCCTATTAACAGCATATGAATATCCTCCTTAG & $\begin{array}{l}\text { mer for } f r a A \text { and } \text { fraBDA } \\
\text { mutagenesis }\end{array}$ \\
\hline BA2513 & GCGCACAAGCCTGCATGATTAATACGTACTCATATGAATATCCTCCTTAG & $a$ island lambda \\
\hline BA2515 & GCCTGCATGATTAATACGTACTGAAATAACTCTGGATCAGCATATGAATATCCTCCTTAG & $\begin{array}{l}\text { reverse primer for fraE lambda red } \\
\text { mutagenesis }\end{array}$ \\
\hline BA2537 & GAGGAAGAAAATGAAAATTAGAGTTTTCATGGCCACCGTGGTGTAGGCTGGAGCTGCTTC & $\begin{array}{l}\text { forward primer for fraE lambda red } \\
\text { mutagenesis }\end{array}$ \\
\hline BA2538 & ATGGATACAAATGATCGAGCAACCCGACAGTAAAAGCGCCGTGTAGGCTGGAGCTGCTTC & $\begin{array}{l}\text { forward primer for fraR and fra } \\
\text { island lambda red mutagenesis }\end{array}$ \\
\hline BA2539 & AATGCTGACATCATACGGTAAACCGTATTTTATCGCCGACCATATGAATATCCTCCTTAG & $\begin{array}{l}\text { reverse primer for fraR lambda red } \\
\text { mutagenesis }\end{array}$ \\
\hline BA2552 & CCTGATGTAATTAATATTCCACTTTCCACATATAGCGGCGCATATGAATATCCTCCTTAG & $\begin{array}{l}\text { forward primer for } f r a B \text { lambda red } \\
\text { mutagenesis }\end{array}$ \\
\hline BA2553 & AGAGGAAAGCATGATGGGTATGAAAGAGACAGTTAGCAATGTGTAGGCTGGAGCTGCTTC & $\begin{array}{l}\text { reverse primer for } f r a B \text { and fraBDA } \\
\text { and fra } B D \text { lambda red mutagenesis }\end{array}$ \\
\hline
\end{tabular}

Table 3. Oligonucleotides.

divided into 15 aliquots and one aliquot was used for each of the following analyses/replicates. Cells were lysed by two cycles of freeze thaw $\left(30 \mathrm{~s}\right.$ at $-20^{\circ} \mathrm{C}$, followed by $90 \mathrm{~s}$ at $\left.37^{\circ} \mathrm{C}\right)$, followed by $120 \mathrm{~s}$ of sonication. Six hundred $\mu \mathrm{l}$ of chilled methanol (Fisher Optima grade, Fisher Scientific) with $16 \mathrm{nmol}\left[{ }^{13} \mathrm{C}\right]-\mathrm{F}$-Asn (internal standard) was added. Five wild-type Salmonella aliquots were spiked with 0, 20, 40, 160 or $320 \mathrm{nmol}$ of 6-P-F-Asp to generate a standard curve. The cell suspension was then vortexed and incubated on ice for $30 \mathrm{~min}$, followed by the addition of $600 \mu \mathrm{l}$ of dichloromethane (Sigma-Aldrich). After being vortexed and centrifuged at 16,200 $\mathrm{g}$ for $10 \mathrm{~min}$ at $4^{\circ} \mathrm{C}$, the upper layer (aqueous phase) was carefully transferred into a new tube with little disturbance of the rest of the mixture. Six hundred $\mu$ l of chilled acetonitrile (Fisher Optima LC/MS grade, Fisher Scientific) was added, followed by vortexing and incubation at $-80^{\circ} \mathrm{C}$ for $2 \mathrm{~h}$. These samples were then centrifuged at $16,200 \mathrm{~g}$ for $20 \mathrm{~min}$ at $4^{\circ} \mathrm{C}$. The supernatants were transferred to new tubes and dried under vacuum (SpeedVac Concentrator, Thermo Scientific). Before mass spectrometry analysis, these dried pellets were resuspended in $900 \mu \mathrm{lmethanol} /$ water, 50\%:50\% with $0.1 \%(\mathrm{v} / \mathrm{v})$ formic acid (LC-MS grade, Thermo Scientific). Samples were introduced into a triple quadrupole mass spectrometer (Waters Xevo TQ-S) by direct infusion at a flow rate of $7 \mu \mathrm{l} / \mathrm{min}$. The mass spectrometer was operated in positive ion electrospray ionization mode (ESI + ) with capillary voltage $3 \mathrm{kV}$, source temperature $150^{\circ} \mathrm{C}$, cone voltage $2 \mathrm{~V}$, cone flow $150 \mathrm{l} / \mathrm{h}$, source offset $2 \mathrm{~V}$, desolvation temperature $350^{\circ} \mathrm{C}$, desolvation gas flow $350 \mathrm{l} / \mathrm{h}$ and nebulizer gas flow 7 bar. The gas flow rate for the collision cell was $0.15 \mathrm{ml} / \mathrm{min}$. While transitions $\mathrm{m} / \mathrm{z} 376 \rightarrow 125$ and $\mathrm{m} / \mathrm{z} 376 \rightarrow 242$ of 6 -P-F-Asp with collision energy $15 \mathrm{eV}$ were selected for quantitation, $\mathrm{m} / \mathrm{z} 301 \rightarrow 216$ of $\left[{ }_{13} \mathrm{C}\right]-\mathrm{F}-$ Asn with collision energy $13 \mathrm{eV}$ was used for normalization. Acquired data were analyzed using Masslynx 4.1. A total of $22 \mathrm{MS} / \mathrm{MS}$ scans of each ion were averaged for the measurement of ion intensity.

Competition experiments. A mutant strain, either fraB::kan HMB206, fraD::kan HMB184 or fra80::kan HMB205, were mixed with the isogenic wild-type strain (JLD1214), respectively, and inoculated by the intragastric (i.g.) route to Swiss Webster mice treated with $20 \mathrm{mg}$ of streptomycin $24 \mathrm{~h}$ prior. Ceca samples were collected five days post-inoculation, homogenized, and plated on XLD kan and XLD cam plates. Antibiotic resistance differentiated the mutant and wild-type strains. The competitive index was calculated as $\mathrm{CI}=$ (cfu mutant recovered/ cfu wild-type recovered)/(cfu mutant input/cfu wild-type input).

Animal assurance. All animal work was performed using protocols approved by our Institutional Animal Care and Use Committee (IACUC; OSU 2009A0035) and in accordance with the relevant guidelines set forth in the PHS "Guide for the Care and Use of Laboratory Animals".

\section{References}

1. Nuccio, S. P. \& Bäumler, A. J. Comparative Analysis of Salmonella Genomes Identifies a Metabolic Network for Escalating Growth in the Inflamed Gut. Mbio. 5, e00929-14-e00929-14 (2014).

2. Scallan, E. et al. Foodborne illness acquired in the United States-major pathogens. Emerging Infect. Dis. 17, 7-15 (2011).

3. Kotloff, K. L. et al. Burden and aetiology of diarrhoeal disease in infants and young children in developing countries (the Global Enteric Multicenter Study, GEMS): a prospective, case-control study. Lancet 382, 209-222 (2013).

4. Majowicz, S. E. et al. The global burden of nontyphoidal Salmonella gastroenteritis. Clin. Infect. Dis. 50, 882-889 (2010).

5. Strugnell, R. A. et al. Salmonella vaccines: lessons from the mouse model or bad teaching? Curr. Opin. Microbiol. 17, 99-105 (2014).

6. Martin, L. B. Vaccines for typhoid fever and other salmonelloses. Current Opinion in Infectious Diseases 25, 489-499 (2012).

7. Gopinath, S., Lichtman, J. S., Bouley, D. M., Elias, J. E. \& Monack, D. M. Role of disease-associated tolerance in infectious superspreaders. Proc. Natl. Acad. Sci. USA 111, 15780-15785 (2014). 
8. Diard, M. et al. Antibiotic Treatment Selects for Cooperative Virulence of Salmonella Typhimurium. Curr. Biol. doi: 10.1016/j. cub.2014.07.028 (2014)

9. Wiström, J. et al. Empiric treatment of acute diarrheal disease with norfloxacin. A randomized, placebo-controlled study. Swedish Study Group. Ann. Intern. Med. 117, 202-208 (1992).

10. Stecher, B. et al. Salmonella enterica serovar typhimurium exploits inflammation to compete with the intestinal microbiota. PLoS Biol. 5, 2177-2189 (2007).

11. Barman, M. et al. Enteric salmonellosis disrupts the microbial ecology of the murine gastrointestinal tract. Infect. Immun. 76, 907-915 (2008)

12. Winter, S. E. et al. Gut inflammation provides a respiratory electron acceptor for Salmonella. Nature 467, 426-429 (2010).

13. Rivera-Chávez, F. \& Bäumler, A. J. The Pyromaniac Inside You: Salmonella Metabolism in the Host Gut. Annu. Rev. Microbiol. doi: 10.1146/annurev-micro-091014-104108 (2015)

14. Winter, S. E. \& Bäumler, A. J. A breathtaking feat: to compete with the gut microbiota, Salmonella drives its host to provide a respiratory electron acceptor. Gut Microbes 2, 58-60 (2011).

15. Thiennimitr, P. et al. Intestinal inflammation allows Salmonella to use ethanolamine to compete with the microbiota. Proc. Natl. Acad. Sci. USA 108, 17480-17485 (2011).

16. Ali, M. M. et al. Fructose-Asparagine Is a Primary Nutrient during Growth of Salmonella in the Inflamed Intestine. PLoS Pathog 10, e1004209 (2014).

17. Hansen, A. L. \& Behrman, E. J. Synthesis of 6-Phosphofructose Aspartic Acid and Some Related Amadori Compounds. Carbohydr. Res 431, 1-5 (2016).

18. Wiame, E., Delpierre, G., Collard, F. \& Van Schaftingen, E. Identification of a pathway for the utilization of the Amadori product fructoselysine in Escherichia coli. J. Biol. Chem. 277, 42523-42529 (2002).

19. Eichner, K., Reutter, M. \& Wittmann, R. Detection of Amadori compounds in heated foods. ACS symposium series (USA) 543, (1994).

20. Anet, E. F. L. J. \& Reynolds, T. M. Chemistry of non-enzymic browning. II. Reactions between Amino Acids, Organic Acids, and sugars in freeze-dried Apricots and Peaches. Aust. J. Chem. 10, 182-191 (1957).

21. Richards, G. R., Patel, M. V., Lloyd, C. R. \& Vanderpool, C. K. Depletion of glycolytic intermediates plays a key role in glucosephosphate stress in Escherichia coli. J. Bacteriol. 195, 4816-4825 (2013).

22. Lee, S. J. et al. Cellular stress created by intermediary metabolite imbalances. Proc. Natl. Acad. Sci. USA 106, 19515-19520 (2009).

23. Irani, M. H. \& Maitra, P. K. Properties of Escherichia coli mutants deficient in enzymes of glycolysis. J. Bacteriol. 132, 398-410 (1977).

24. Englesberg, E. et al. L-Arabinose-sensitive, L-ribulose 5-phosphate 4-epimerase-deficient mutants of Escherichia coli. J. Bacteriol. 84, $137-146$ (1962).

25. Yarmolinsky, M. B., Wiesmeyer, H., Kalckar, H. M. \& Jordan, E. Hereditary defects in galactose metabolism in Escherichia coli mutants, II. Galactose-induced sensitivity. Proc. Natl. Acad. Sci. USA 45, 1786-1791 (1959).

26. Englesberg, E. \& Baron, L. S. Mutation to L-rhamnose resistance and transduction to L-rhamnose utilization in Salmonella typhosa. J. Bacteriol. 78, 675-686 (1959).

27. Bobrovskyy, M. \& Vanderpool, C. K. The small RNA SgrS: roles in metabolism and pathogenesis of enteric bacteria. Front Cell Infect Microbiol 4, 61 (2014).

28. Lee, S. J., Trostel, A. \& Adhya, S. Metabolite changes signal genetic regulatory mechanisms for robust cell behavior. MBio 5, e00972-13 (2014)

29. Miller, J. H. Experiments in molecular genetics. (Cold Spring Harbor Laboratory, 1972).

30. Price-Carter, M., Tingey, J., Bobik, T. A. \& Roth, J. R. The Alternative Electron Acceptor Tetrathionate Supports B12-Dependent Anaerobic Growth of Salmonella enterica Serovar Typhimurium on Ethanolamine or 1,2-Propanediol. J. Bacteriol. 183, 2463-2475 (2001).

31. Datsenko, K. A. \& Wanner, B. L. One-step inactivation of chromosomal genes in Escherichia coli K-12 using PCR products. Proc. Natl. Acad. Sci. USA 97, 6640-6645 (2000).

32. Bradford, M. M. A rapid and sensitive method for the quantitation of microgram quantities of protein utilizing the principle of protein-dye binding. Analytical Biochemistry 72, 248-254 (1976).

33. Brown, R. N. et al. A Comprehensive Subcellular Proteomic Survey of Salmonella Grown under Phagosome-Mimicking versus Standard Laboratory Conditions. Int J Proteomics 2012, Article ID 123076 (2012).

34. Cherepanov, P. P. \& Wackernagel, W. Gene disruption in Escherichia coli: TcR and KmR cassettes with the option of Flp-catalyzed excision of the antibiotic-resistance determinant. Gene 158, 9-14 (1995).

\section{Acknowledgements}

We thank Prof. Valeri Mossine (University of Missouri) for suggesting a strategy to overcome a crippling solubility problem in the synthesis of Amadori compounds. We thank Susan Egan (University of Kansas) and Diana Downs (University of Georgia) for helpful discussions of sugar-phosphate stress. This work was supported by NIH NIAID grant 1R01AI116119.

\section{Author Contributions}

A.S.-D., H.M.B., A.S., J.W., A.J.B., M.M.A., C.S. and E.J.B. performed the experiments. V.H.W., V.G., E.J.B. and B.M.M.A. designed and supervised the experiments. A.S.-D., A.S., J.W., V.H.W., V.G., E.J.B. and B.M.M.A. wrote the manuscript. All authors reviewed the manuscript.

\section{Additional Information}

Supplementary information accompanies this paper at http://www.nature.com/srep

Competing financial interests: The authors declare no competing financial interests.

How to cite this article: Sabag-Daigle, A. et al. A metabolic intermediate of the fructose-asparagine utilization pathway inhibits growth of a Salmonella fraB mutant. Sci. Rep. 6, 28117; doi: 10.1038/srep28117 (2016). unless indicated otherwise in the credit line; if the material is not included under the Creative Commons license, users will need to obtain permission from the license holder to reproduce the material. To view a copy of this license, visit http://creativecommons.org/licenses/by/4.0/ 